\title{
Tame Köthe Sequence Spaces are Quasi-Normable
}

\author{
by \\ Krzysztof PISZCZEK \\ Presented by Czestaw BESSAGA
}

Summary. We show that every tame Fréchet space admits a continuous norm and that every tame Köthe sequence space is quasi-normable.

1. Introduction. First we recall definitions and basic properties of the above mentioned classes of spaces. Let $X$ be a Fréchet space with the topology defined by an increasing sequence $\left(\|\cdot\|_{n}\right)_{n \in \mathbb{N}}$ of seminorms. We call $X$ tame if the following condition holds: there is an increasing function $S: \mathbb{N} \rightarrow \mathbb{N}$ such that for every continuous linear operator $T: X \rightarrow X$ there is a natural $k_{0}$ such that for every $k \geq k_{0}$ there is a constant $C_{k}$ such that

$$
\|T x\|_{k} \leq C_{k}\|x\|_{S(k)} \quad \text { for every } x \in X .
$$

This class of spaces was defined by D. Vogt and E. Dubinsky in [3]. They proved that in a tame infinite type power series space every complemented subspace has a basis. For other papers related to the notion of tameness see [7]-[9]. It is known that every finite type power series space is tame (see [10, Lemma 5.1]). The aim of this paper is to analyze which Köthe sequence spaces are tame.

We call $X$ quasi-normable if for every 0-neighbourhood $U$ there exists another 0 -neighbourhood $V$ such that for every $\varepsilon>0$ we can find a bounded set $B$ in $X$ such that

$$
V \subset \varepsilon U+B .
$$

The class of quasi-normable spaces was introduced by A. Grothendieck in [4]. See also [2], [6]. By $L(X)$ we denote the linear space of all continuous linear

2000 Mathematics Subject Classification: Primary 46A61; Secondary 46A45.

Key words and phrases: tame Fréchet space, Köthe sequence space, quasi-normable, continuous norm. 
operators acting on $X$. For any operator $A \in L(X)$ we define

$$
\sigma_{A}(k)=\inf \left\{n \in \mathbb{N}: \sup _{\|x\|_{n} \leq 1}\|A x\|_{k}<\infty\right\} .
$$

Let $I$ be an arbitrary index set and $A=\left(a^{n}\right)_{n \in \mathbb{N}}$ a sequence of nonnegative functions defined on $I$ with the property that $a_{i}^{n} \leq a_{i}^{n+1}$ for all $n \in \mathbb{N}, i \in I$. Let us recall that for $1 \leq p<\infty$ a Köthe sequence space is defined as follows:

$$
\lambda_{p}(I, A)=\left\{x=\left(x_{1}, x_{2}, \ldots\right):\|x\|_{k}:=\left(\sum_{i \in I}\left(a_{i}^{k}\left|x_{i}\right|\right)^{p}\right)^{1 / p}<\infty \forall k \in \mathbb{N}\right\}
$$

and

$$
\lambda_{\infty}(I, A)=\left\{x=\left(x_{1}, x_{2}, \ldots\right):\|x\|_{k}:=\sup _{i \in I} a_{i}^{k}\left|x_{i}\right|<\infty \forall k \in \mathbb{N}\right\}
$$

(see $[5,27])$. For other notions from functional analysis used in this paper see [5].

\section{Preliminary results}

LEMMA 1. The space $\omega$ of all sequences is not tame.

Proof. Recall that

$$
\omega=\left\{x=\left(x_{1}, x_{2}, \ldots\right):\|x\|_{k}:=\max _{j \leq k}\left|x_{j}\right|<\infty\right\} .
$$

Let $S: \mathbb{N} \rightarrow \mathbb{N}$ be an arbitrary increasing function and let $A: \omega \rightarrow \omega$ be an operator defined as

$$
A\left(\left(x_{j}\right)_{j \in \mathbb{N}}\right)=\left(x_{S(j+1)}\right)_{j \in \mathbb{N}} .
$$

Let

$$
\begin{array}{r}
x^{(n)}=(0, \ldots, 0, n, 0, \ldots) . \\
\downarrow \\
\text { place } S(k+1)
\end{array}
$$

Then $\left\|A x^{(n)}\right\|_{k}=n$ and $\left\|x^{(n)}\right\|_{S(k)}=0$. Therefore there is no constant $C$ such that $\|A x\|_{k} \leq C\|x\|_{S(k)}$ for all $x \in \omega$, which proves that $\omega$ is not tame.

Lemma 2. Tameness is inherited by complemented subspaces.

Proof. Let $P: E \rightarrow X$ be a projection. If $A$ is a continuous linear operator on $X$ then the operator $A \circ P: E \rightarrow X$ is an element of $L(E)$. Thus

$$
\|A x\|_{k}=\|A \circ P x\|_{k} \leq C_{k}\|x\|_{\sigma_{A P}(k)}
$$

and $\sigma_{A}(k) \leq \sigma_{A P}(k)$. If $\sigma_{A P}(k) \leq S(k)$ then $\sigma_{A}(k) \leq S(k)$ and thus if $E$ is tame then $X$ is tame as well. 
Let $\phi: \mathbb{N} \rightarrow \mathbb{N}$ be an arbitrary increasing function and define spaces of linear continuous operators

$$
\begin{aligned}
L_{\phi}(X) & =\left\{A \in L(X): \forall k \in \mathbb{N} \exists C_{k} \forall x \in X \quad\|A x\|_{k} \leq C_{k}\|x\|_{\phi(k)}\right\}, \\
L_{\phi, n}(X) & =\left\{A \in L(X): \forall k \geq n \exists C_{k} \forall x \in X \quad\|A x\|_{k} \leq C_{k}\|x\|_{\phi(k)}\right\} .
\end{aligned}
$$

If we put

$$
\|A\|_{\phi(i), i}=\sup _{\|x\|_{\phi(i)} \leq 1}\|A x\|_{i}
$$

then $L_{\phi}(X)$ and $L_{\phi, n}(X)$ are Fréchet spaces with the sequences of seminorms defined as $\|\cdot\|_{m}=\max _{1 \leq i \leq m}\|\cdot\|_{\phi(i), i}$ and $\|\cdot\|_{m}=\max _{n \leq i \leq m}\|\cdot\|_{\phi(i), i}$, respectively. Only completeness needs a comment. If $\left(A_{p}\right)_{p}$ is a Cauchy sequence in $L_{\phi}(X)$ then for every $x \in X$ the sequence $\left(A_{p} x\right)_{p}$ is a Cauchy sequence in the complete space $X$. This means that for the operator $A x=\lim _{p \rightarrow \infty} A_{p} x$ we have

$$
\forall k \in \mathbb{N} \exists P \in \mathbb{N}: \quad\left\|\left(A-A_{P}\right) x\right\|_{k} \leq\|x\|_{\phi(k)} .
$$

This implies that $\|A x\|_{k} \leq\left(C_{k}^{P}+1\right)\|x\|_{\phi(k)}=D_{k}\|x\|_{\phi(k)}$ for all $k$, which shows that $A \in L_{\phi}(X)$. The proof in the case of $L_{\phi, n}(X)$ is the same.

LEMMA 3. In every tame Fréchet space $X$ the following condition holds: there exists $\psi: \mathbb{N} \rightarrow \mathbb{N}$ such that for any $\phi: \mathbb{N} \rightarrow \mathbb{N}$ there exists $k \in \mathbb{N}$ such that for all $m \geq k$ there are $n \in \mathbb{N}$ and a constant $C_{m}>0$ such that

$$
\forall x^{*} \in X^{*}, y \in X: \quad \max _{k \leq l \leq m}\left\|x^{*}\right\|_{\psi(l)}^{*}\|y\|_{l} \leq C_{m} \max _{1 \leq p \leq n}\left\|x^{*}\right\|_{\phi(p)}^{*}\|y\|_{p},
$$

where $\left\|x^{*}\right\|_{m}^{*}=\sup _{\|x\|_{m} \leq 1}\left|x^{*}(x)\right|$.

Proof. If the space $X$ is tame with the function $\psi$ then every continuous linear operator is an element of a certain $L_{\psi, k}$ so we may write $L(X)=\bigcup_{k \in \mathbb{N}} L_{\psi, k}(X)$. If we now endow the space $L(X)$ with the topology of pointwise convergence then for every increasing function $\phi: \mathbb{N} \rightarrow \mathbb{N}$ we obtain the following diagram where the arrows represent continuous linear mappings:

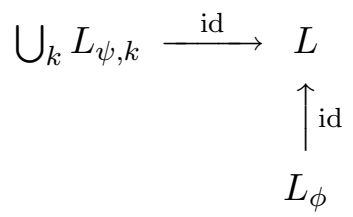

The continuity of the horizontal arrow comes from the following argument: for every 0-neighbourhood $U\left(0, x_{1}, \ldots, x_{n}, k, \varepsilon\right)=\{A \in L(X): \forall 1 \leq i \leq n$ $\left.\left\|A x_{i}\right\|_{k}<\varepsilon\right\}$ in $L$ we define a 0-neighbourhood $V=\left\{A \in L_{\phi}:\|A\|_{k}<\right.$ $\varepsilon / M\}$ in $L_{\phi}$, where $M=\max _{1 \leq i \leq n}\left\|x_{i}\right\|_{k}$. As is easily seen, $\operatorname{id}(V) \subset U$. The continuity of the vertical arrow is proved similarly. Using Grothendieck's Factorization Theorem [5, 24.33] we find a natural number $k$ such that $L_{\phi}$ 
embeds continuouosly in $L_{\psi, k}$. In other words in the tame Fréchet space the following holds:

$$
\begin{array}{r}
\exists \psi \nearrow \infty \forall \phi \nearrow \infty \exists k \forall m \geq k \exists n, C_{m} \forall T \in L_{\phi}(X): \\
\max _{k \leq l \leq m}\|T\|_{\psi(l), l} \leq C_{m} \max _{1 \leq p \leq n}\|T\|_{\phi(p), p} .
\end{array}
$$

In particular, for one-dimensional operators $T, T x=x^{*}(x) y, x^{*} \in X, y \in X$, we get (1).

Lemma 4. Let $\lambda_{p}(I, A)$ be an arbitrary Köthe sequence space. If it is not quasi-normable then, without loss of generality, we may assume that $A$ satisfies the following conditions: $a_{i}^{1}=1$ for all $i$, and for every natural number $m$ there exists an index subset $J_{m}=\{i(m, j): j \in \mathbb{N}\}$ such that

$$
\sup _{j} a_{i(m, j)}^{m}=c_{m}<\infty \quad \text { and } \quad \lim _{j} a_{i(m, j)}^{m+1}=\infty .
$$

Proof. From [2, Th. 17] it follows that if $\lambda_{p}(I, A)$ is not quasi-normable then

$\exists n \forall m \geq n \exists J \subset I: \quad \inf _{i \in J} \frac{a_{i}^{n}}{a_{i}^{m}}>0 \quad$ and $\quad \inf _{i \in J} \frac{a_{i}^{n}}{a_{i}^{k}}=0 \quad$ for some $k(m) \geq m$.

Firstly, we may assume that $n=1$ and $a_{i}^{1}=1$ for all $i$ (by dividing by $a_{i}^{1}$ ). Secondly, every set $J_{m}$ is infinite so we may write $J_{m}=\{i(m, j): j \in \mathbb{N}\}$. Finally, omitting rows of the matrix $A$ suitably, numbers $k(m)$ can be chosen as $k(m)=m+1$ for $m \in \mathbb{N}$.

\section{Main results}

Proposition 5. Every tame Fréchet space has a continuous norm.

Proof. If the space does not admit a continuous norm then from [1, Lemmas 1 and 2] it contains $\omega$ as a complemented subspace; but then from our assumption and Lemma 2, $\omega$ is tame, which contradicts Lemma 1.

THEOREM 6. Tame Köthe sequence spaces are quasi-normable.

Proof. By Proposition 5 we may assume that $a_{i}^{k}>0$ for all $i \in I, k \in \mathbb{N}$. Suppose that $\lambda_{p}(I, A)$ is a tame Köthe space which is not quasi-normable. Using Lemma 3 we may write

$$
\left\|x^{*}\right\|_{\psi(k)}^{*}\|y\|_{k} \leq C_{k} \max _{1 \leq p \leq n}\left\|x^{*}\right\|_{\phi(p)}^{*}\|y\|_{p} .
$$

Without losing of generality we may assume that $n \geq k$. For all $j, v \in \mathbb{N}$ define

$$
x_{v}^{*} x=x_{i(\phi(k-1), v)} \quad \text { and } \quad y_{j}=e_{i(k-1, j)},
$$

where $x_{i}$ denotes the $i$ th coordinate of the vector $x, e_{i}$ is the $i$ th vector of 
the standard basis, and $i(k, j)$ denotes the index of number $j$ from the index set $J_{k}$. Since $\left\|y_{j}\right\|_{p}=a_{i(k-1, j)}^{p}$ and $\left\|x_{v}^{*}\right\|_{l}^{*}=\left(a_{i(\phi(k-1), v)}^{l}\right)^{-1}$, we obtain for all $j, v \in \mathbb{N}$ the inequality

$$
\frac{a_{i(k-1, j)}^{k}}{a_{i(\phi(k-1), v)}^{\psi(k)}} \leq C_{k} \max _{1 \leq p \leq n} \frac{a_{i(k-1, j)}^{p}}{a_{i(\phi(k-1), v)}^{\phi(p)}} .
$$

The function $\phi$ has been arbitrary so far but from now on we choose $\phi(k-1)$ $=\psi(k)$. Without loss of generality we may assume that $\psi$ is strictly increasing, which, combined with Lemma 4, gives us

$$
a_{i(\phi(k-1), v)}^{\psi(k)}=a_{i(\phi(k-1), v)}^{\phi(k-1)} \leq c_{\phi(k-1)}
$$

for all $v$ and

$$
a_{i(k-1, j)}^{k} \underset{j \rightarrow \infty}{\longrightarrow} \infty .
$$

Equivalently we may write

$$
\frac{1}{c_{\phi(k-1)}} a_{i(k-1, j)}^{k} \leq \frac{a_{i(k-1, j)}^{k}}{a_{i(\phi(k-1), v)}^{\psi(k)}} .
$$

The estimates of the right hand side of (4) will be divided into two cases. If $p \leq k-1$ then

$$
a_{i(k-1, j)}^{p} \leq a_{i(k-1, j)}^{k-1} \leq c_{k-1} \quad \text { and } \quad a_{i(\phi(k-1), v)}^{\phi(p)} \geq a_{i(\phi(k-1), v)}^{1}=1,
$$

for all $j, v$. If $p \geq k$ then also $\phi(p) \geq \phi(k) \geq \phi(k-1)+1$, which leads to

$$
a_{i(\phi(k-1), v)}^{\phi(p)} \geq a_{i(\phi(k-1), v)}^{\phi(k-1)+1} \underset{v \rightarrow \infty}{\longrightarrow} \infty
$$

and

$$
a_{i(k-1, j)}^{p} \geq a_{i(k-1, j)}^{k} \underset{j \rightarrow \infty}{\longrightarrow} \infty .
$$

This implies that for every natural number $j$ there is an index $v_{j} \in \mathbb{N}$ depending on $k$ but not on $p$ such that $a_{i\left(\phi(k-1), v_{j}\right)}^{\phi(p)} \geq a_{i(k-1, j)}^{p}$. If we now extract from $\left\{x_{v}^{*}\right\}_{v=1}^{\infty}$ the subsequence $\left(x_{v_{j}}^{*}\right)_{j \in \mathbb{N}}$ then we obtain the inequality

$$
\max _{1 \leq p \leq n} \frac{a_{i(k-1, j)}^{p}}{a_{i\left(\phi(k-1), v_{j}\right)}^{\phi(p)}} \leq \max \left\{c_{k-1}, 1\right\}=d_{k} .
$$

Combining the inequalities (4), (6) and (7) we finally get

$$
a_{i(k-1, j)}^{k} \leq C_{k} c_{\phi(k-1)} d_{k}<\infty \quad \text { for all } j ;
$$

but, by (5), $\lim _{j} a_{i(k-1, j)}^{k}=\infty$, a contradiction. This completes the proof. 


\section{References}

[1] C. Bessaga and A. Pełczyński, On a class of $B_{0}$-spaces, Bull. Acad. Polon. Sci. Cl. III 5 (1957), 375-377.

[2] K. D. Bierstedt and J. Bonet, Some aspects of the modern theory of Fréchet spaces, RACSAM Rev. R. Acad. Cienc. Exactas Fis. Nat. Serie A Mat. 97 (2003), 159-188.

[3] E. Dubinsky and D. Vogt, Complemented subspaces in tame power series spaces, Studia Math. 93 (1989), 71-85.

[4] A. Grothendieck, Sur les espaces (F) et (DF), Summa Brasil. Math. 3 (1954), 57122.

[5] R. Meise and D. Vogt, Introduction to Functional Analysis, Clarendon Press, Oxford, 1997.

[6] -, A characterization of the quasi-normable Fréchet spaces, Math. Nachr. 122 (1985), 141-150.

[7] K. Nyberg, Tameness of pairs of nuclear power series spaces and related topics, Trans. Amer. Math. Soc. 283 (1984), 645-660.

[8] M. Poppenberg and D. Vogt, Construction of standard exact sequences of power series spaces, Studia Math. 112 (1995), 229-241.

[9] - A tame splitting theorem for exact sequences of Fréchet spaces, Math. Z. 219 (1995), 141-161.

[10] D. Vogt, Eine Charakterisierung der Potenzreihenräume von endlichem Typ und ihre Folgerungen, Manuscripta Math. 37 (1982), 269-301.

Krzysztof Piszczek

Faculty of Mathematics and Computer Science

Adam Mickiewicz University

Umultowska 87

61-614 Poznań, Poland

E-mail: kpk@amu.edu.pl 